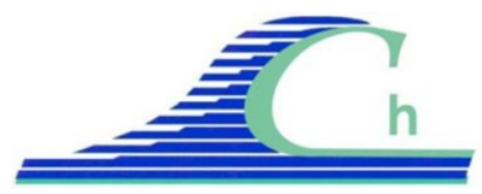

XII ${ }^{\text {ìmes }}$ Journées Nationales Génie Côtier - Génie Civil

Cherbourg, 12-14 juin 2012

DOI:10.5150/jngcgc.2012.091-R @ Editions Paralia CFL

disponible en ligne - http://www.paralia.fr - available online

\title{
La viabilité économique des énergies marines comme condition nécessaire de leur développement en France et à l'international
}

\author{
Antoine RABAIN ${ }^{1}$, Yann-Hervé DE ROECK ${ }^{2}$
}

\section{INDICTA, Responsable du pôle Energies, Ressources \& Technologies Vertes} 18 rue Horace Vernet, 92130 Issy-les-Moulineaux, France.

antoine.rabain@indicta.com

2. FRANCE ENERGIES MARINES, Directeur Général, Technopôle Brest-Iroise, 29280 Plouzané, France.

yhdr@france-energies-marines.org

\section{Résumé :}

Les énergies marines sont considérées par un nombre croissant d'acteurs politiques et économiques français comme des filières stratégiques permettant de répondre aux exigences énergétiques et climatiques de court, moyen et long termes. Les conditions de démarrage et les perspectives de développement de ces marchés émergents dépendent fondamentalement des spécificités et du potentiel de la ressource exploitée, ainsi que de la maturité des technologies. Aujourd'hui, les incertitudes se concentrent sur l'attractivité socio-économique des projets et la définition de subventions publiques adaptées, qui conditionnent la dynamique de pénétration des technologies.

Dans cette contribution, les résultats de modélisation sur l'évolution des coûts complets de production des différentes technologies, dont les hypothèses de base ont été affinées avec un ensemble d'industriels français de référence, ont permis de dresser les conditions de démarrage de chacun des segments des énergies marines, ainsi que d'établir une prospective de ces marchés en France et au niveau mondial d'ici à 2030.

Les deux segments technologiquement les plus matures, l'éolien offshore et l'hydrolien, sont en mesure de se développer de façon imminente, tandis que le houlomoteur est encore en phase de structuration technologique, et repose à ce stade sur des conditions de démarrage similaires à celles que connaît l'hydrolien, avec des technologies permettant de valoriser l'énergie des vagues à proximité des côtes. Le déficit de compétitivité structurel de l'Energie Thermique des Mers vis-à-vis des autres énergies marines est quant à lui compensé par ses caractéristiques énergétiques (notamment la production d'électricité de base) et sa pertinence en zone insulaire, où les coûts de production sont significativement plus élevés que dans les territoires continentaux.

\section{Mots-clés :}

Énergies marines - Eolien offshore (posé et flottant) - Hydrolien - Houlomoteur Energie Thermique des Mers (ETM) - Coûts de production - Prospective de marchés 


\section{Introduction et positionnement de la problématique}

1.1 Enjeux énergétiques et climatiques - Criticité du développement des énergies marines en France et à l'international

La double pression que constituent la raréfaction des énergies fossiles et les changements climatiques d'origine anthropique implique une révolution énergétique, et notamment le développement accéléré de systèmes de production d'électricité non carbonée. Devant l'incapacité des technologies les plus matures (hydroélectricité, éolien terrestre, solaire photovoltaïque, biomasse, ...) à répondre seules aux exigences fixées à horizon 2050 par le GIEC (Groupe d'experts Intergouvernemental sur l'Evolution du Climat), les énergies marines représentent - avec le solaire thermique à concentration l'un des leviers les plus stratégiques au sein des technologies n'ayant pas encore atteint le stade commercial, et sont considérées par un nombre croissant d'industriels de l'énergie comme les marchés offrant les perspectives de croissance les plus prometteuses sur le long terme.

Si l'historique de développement des systèmes permettant l'exploitation des énergies marines remonte à plusieurs décennies, les conditions économiques, politiques et technologiques sont aujourd'hui réunies pour anticiper des investissements massifs sur l'ensemble de ces marchés d'innovation, et en premier lieu au sein des grands pays de l'économie maritime. Le développement des premiers prototypes de taille réduite et surtout l'arrivée récente des premiers démonstrateurs - ou prototypes à échelle 1:1 conduisent aujourd'hui à penser qu'un développement industriel pourrait commencer à se concrétiser vers 2015 et tendre vers une structuration de nouvelles filières industrielles à fort potentiel d'exportation dans les quinze prochaines années. La quasitotalité des pionniers cherchant aujourd'hui à contribuer au démarrage du marché des énergies marines se trouve sur le territoire européen. Parmi eux, la France possède des atouts considérables, avec un potentiel de valorisation énergétique parmi les plus importants au niveau mondial, notamment grâce à l'étendue de sa Zone Economique Exclusive, qui dépasse les onze millions de $\mathrm{km}^{2}$ (deuxième au monde après les EtatsUnis) et à une présence géostratégique sur l'ensemble des eaux de la planète.

1.2 La réalisation des ambitions de la France dans les énergies marines passe par le regroupement des forces multipartites et par le soutien des politiques publiques

La création d'Instituts d'Excellence pour les Energies Décarbonnées (IEED) au sein des Investissements d'Avenir constitue pour la France une opportunité de regrouper l'ensemble des parties prenantes critiques -publiques et privées- afin d'optimiser les chances de succès de la filière nationale des énergies marines et de prétendre à terme à un rôle de premier plan à l'international. Coordonné par l'IFREMER, France Energies Marines concourt au statut d'IEED depuis 2010, et a produit, dans le cadre de sa candidature, un certain nombre de travaux évaluant les investissements nécessaires pour 


\section{XII ${ }^{\text {èmes }}$ Journées Nationales Génie Côtier - Génie Civil \\ Cherbourg, 12-14 juin 2012}

permettre une montée en puissance des industriels nationaux, en phase avec la dynamique de développement observée à l'étranger. La validation du soutien national dans ce mode d'organisation innovant dépendait, parmi d'autres exigences, d'une démonstration de l'évolution de l'attractivité économique de l'ensemble de la filière et de la viabilité économique du marché des énergies marines à moyen et long termes.

Au-delà des subventions publiques à l'investissement, qui sont appropriées lors des phases de validation des technologies, ce sont davantage les prix de rachat préférentiel de l'électricité produite qui guident le soutien des politiques publiques lorsque les technologies entrent en phase de commercialisation. Alors que ces niveaux de subvention sont directement liés aux coûts de production des systèmes, les derniers travaux de référence menés au niveau national consistaient en une prospective à 2030 coordonnée par l'IFREMER (PAILLARD et al., 2007) et aux travaux de l'initiative IPANEMA (2009). Une actualisation détaillée et soutenue par l'ensemble des acteurs pionniers, a été considérée comme indispensable pour préciser l'évolution de l'attractivité économique de ces technologies. Le manque de consensus apparent laissait trop d'incertitudes, aux yeux des décideurs publics, pour engager un soutien durable au développement des énergies marines.

Cet article rend compte des travaux réunissant l'ensemble des données de référence faisant apparaître les seuils d'acceptabilité économique des segments des énergies marines lors de leur entrée respective en phase de commercialisation, ainsi que des résultats de l'analyse prospective réalisée au niveau mondial par la modélisation des coûts complets de production des systèmes sur la période $\{2010 ; 2030\}$.

\section{Méthodologie}

\subsection{Définition d'un périmètre adapté pour l'analyse des segments les plus pertinents}

Les systèmes permettant d'exploiter les énergies marines font référence à un ensemble de technologies dont la maturité diffère (figure 1) et dont les fondamentaux des marchés associés permettent d'identifier des perspectives d'évolution différentes.

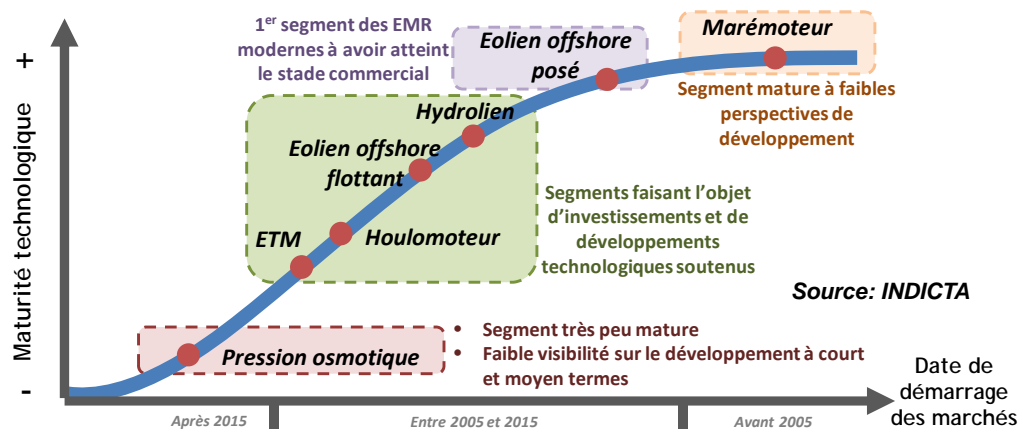

Figure 1. Degré de maturité relatif aux sept segments des énergies marines (courbe en S) et définition du périmètre d'analyse. 


\section{Thème 5 - Énergies marines}

Le marémoteur est le segment historique des énergies marines sur lequel la France s’est positionnée en tant que pays pionnier dans les années 60. Le segment a bénéficié du savoir-faire reconnu des acteurs français de l’hydroélectricité (génie civil, turbines de barrages, ...), mais suscite aujourd'hui un faible intérêt des acteurs et des politiques, principalement pour des considérations environnementales. Avec les progrès réalisés sur les turbines de basse-chute, l'aménagement de zones portuaires déjà fortement anthropisées devient cependant possible.

Sur l'éolien offshore posé, segment dont la maturité des technologies est la plus élevée, la France dispose de fortes ambitions, avec un objectif de puissance installée de 6 GW à l'horizon 2020, soit environ deux fois le parc actuel mondial. Dans un tel contexte, les industriels français renforcent leurs positions sur le marché grâce à des stratégies de croissance externe afin de tirer parti du contexte de développement national, mais aussi à des fins d'internationalisation.

L'éolien offshore flottant, en plus des conditions d'accès facilitées pour les turbinistes, bénéficiera pour la conception du flotteur d'une valorisation de savoir-faire issus des univers de la construction navale et des plateformes pétrolières et gazières. Le marché potentiel à terme est considérable, et les zones dépourvues d'une bathymétrie favorable aux technologies sur fondation, constitueront les pays cibles des pionniers ; à ce titre, la France présente une forte attractivité, en particulier en mer Méditerranée.

Les technologies d'hydroliennes bénéficient des savoir-faire issus de l'univers des turbines (hydroélectricité et propulsion navale) ; de plus, le segment présente une ressource localisée, bien identifiée et dense, permettant d'entrevoir une croissance rapide et soutenue du marché, ce qui incite les industriels à considérer le segment comme le plus attractif des énergies marines à court terme (hors éolien offshore posé). La France représente le deuxième marché potentiel européen, avec une capacité d'environ 3 GW (pour un total de 11 GW en Europe).

Bien que bénéficiant de ressources naturelles considérables, le houlomoteur et l'ETM font appel à des principes technologiques moins éprouvés, ce qui conduit à retenir une visibilité sur la dynamique de pénétration de ces systèmes plus réduite. On anticipe cependant un démarrage imminent d'un sous-segment du marché du houlomoteur, dont la ressource est proche des côtes et exploitée par des systèmes spécifiques dits "nearshore". Sur l'ETM, le français DCNS et l'américain Lockheed Martin sont les seuls industriels au monde à pouvoir proposer d'ici cinq années une centrale ETM commerciale, les DOM COM constituant une voie d'accès stratégique pour l'industriel français. A ce titre la France représente l'un des pays les plus attractifs au monde pour ce type de technologies.

Sur la pression osmotique enfin, on observe un développement aujourd'hui à peine entamé, et la maturité technologique est encore trop faible pour anticiper une contribution importante de ce type de technologies avant 2030. 


\section{XII ${ }^{\text {èmes }}$ Journées Nationales Génie Côtier - Génie Civil \\ Cherbourg, 12-14 juin 2012}

Ainsi, n’ont été retenus dans le périmètre d'analyse que les cinq segments considérés à ce jour comme susceptibles d'occuper une place centrale dans les investissements français et mondiaux des dix prochaines années dédiés aux énergies marines, soient: l'éolien offshore posé et flottant, l'hydrolien, le houlomoteur et l'ETM.

2.2 Modélisation de l'évolution des coûts complets de production des différents segments retenus sur la période $\{2010 ; 2030\}$

Les conditions de viabilité économique des énergies marines à court, moyen et long termes ont été estimées par une analyse de l'évolution des coûts de production ( $€ / \mathrm{MWh})$ des systèmes. Ce travail de modélisation est issu de la méthode dite des "coûts complets hors externalités", qui intègre un certain nombre de segments de coûts dans le cycle de vie des projets commerciaux. Cette méthodologie, usuellement retenue dans les évaluations économiques des systèmes de production électrique, qu'ils soient conventionnels ou émergents, rend compte des coûts moyens de production d'un système sur l'ensemble de sa durée de vie. L'ensemble des données de base retenues dans cette modélisation a été défini lors de nombreux échanges confidentiels avec les industriels français les plus avancés sur chacun des segments, et a été anonymisé et moyenné par la suite.

La première variable sensible du modèle est le coût d'investissement ( $€ / \mathrm{MW})$ : il inclut le coût de fabrication et d'installation du système, ainsi que les exigences de rentabilité des investisseurs ; le taux d'actualisation (qui rend compte de la rémunération des capitaux engagés à l'investissement) communément retenu sur le secteur de la production d'électricité renouvelable atteint en général 8 à 10\%. Si les coûts de raccordement au réseau terrestre sont également inclus dans le coût d'investissement, le coût de démantèlement du système n’a pas été renseigné, car il reste aujourd'hui difficile d'estimer ce chiffre avec une précision acceptable.

La deuxième variable permettant la modélisation des coûts complets de production rend compte des coûts d'opération et de maintenance des systèmes : ils ont été renseignés dans le modèle par un pourcentage du coût d'investissement, et varient entre 6 et $8 \%$, en fonction de la complexité des technologies et des zones d'exploitation.

Une autre variable critique du modèle rend compte de la performance des technologies : elle est ici valorisée par le facteur de charge de chaque système. Ce dernier fait référence au nombre d'heures de fonctionnement dans une année à puissance nominale maximale. Autrement dit, il détermine le productible annuel d'un système d'une puissance de $1 \mathrm{MW}$, qui produira à un rendement de $100 \%$ un certain nombre de MWh sur les 8766 heures d'une année pleine.

Enfin, la durée de vie des installations constitue la dernière variable du modèle : nous avons retenu la durée de vie minimale des installations sur les marchés connexes de type éolien terrestre ou solaire, à savoir 20 ans, qui correspond à une limite basse vis-à-vis des exigences des énergéticiens. 


\section{Thème 5 - Énergies marines}

Le coût des externalités, notamment les impacts de la pénétration de systèmes de production intermittente sur les investissements dans les réseaux (problématique commune à de nombreuses énergies renouvelables), n’a pas été intégré ici à la méthodologie qui visait surtout à établir une base de référence sur le coût réel des technologies et sur leurs évolutions dès la phase de commercialisation entamée.

\section{Résultats de la modélisation des coûts complets de production sur la période} $\{2010 ; 2030\}$

Chacun des segments analysés se caractérise par des perspectives de croissance différentes, où la dynamique d'évolution des coûts complets de production diffère, même si tous sont voués a diminuer rapidement dans les 20 prochaines années.

Le segment de l'éolien offshore posé (tout juste entré depuis fin 2008 en phase de croissance soutenue au Nord de l'Europe) présente aujourd'hui la meilleure attractivité économique des énergies marines (hors marémoteur) avec un coût complet de production qui oscille entre 150 et $200 € / M W h$ selon les sites. Après 2025 et le déploiement massif des technologies, les coûts complets de production sur les meilleurs sites pourraient atteindre la limite symbolique des $100 € / \mathrm{MWh}$.

La dépendance du marché de l'éolien flottant avec ceux de l'éolien terrestre et de l'éolien offshore posé incite les développeurs à reconsidérer les architectures matures des turbines d'éoliennes, et cherchent à valider de nouveaux concepts, qui permettent de réduire les coûts d'investissement et d'opération par l'optimisation du dimensionnement du flotteur et de la phase d'intégration du système. Plus généralement, l'éolien flottant bénéficiera des retours d'expérience des industriels présents sur le marché des éoliennes posées, et les développeurs de nouvelles architectures se focalisent sur un objectif de coût complet de production de l'ordre de 180 à 200 €/MWh pour garantir le démarrage du marché et l'attractivité des technologies au sein des pays les plus sensibles à ce type de technologies. A plus long terme, et selon les arbitrages technologiques gagnants qui seront identifiés dans les cinq prochaines années, le potentiel élevé de renforcement de l'attractivité économique des systèmes permet d'anticiper une croissance soutenue dès 2020, lorsque les coûts complets de production des fermes atteindront le seuil des $150 € / M W h$. On identifie enfin des ruptures technologiques possibles permettant de viser plus rapidement, et avant 2030, le seuil symbolique des $100 € / \mathrm{MWh}$ et ainsi entrevoir une compétitivité renforcée de l'éolien flottant sur l'éolien offshore posé.

Les quelques dizaines de démonstrateurs d’hydroliennes d’ores et déjà validés dans le monde permettent d'anticiper un démarrage imminent du marché. On évalue les coûts complets de production des premières fermes pré-commerciales entre 200 et 250 €/MWh. Le premier GW de capacité cumulée installée permet d'anticiper une baisse significative des coûts complets de production et de viser, compte tenu des perspectives de croissance soutenue du marché, un seuil de $150 € / \mathrm{MWh}$ avant 2020. A moyen terme, le seuil de la dizaine de GW (soit environ 10000 hydroliennes en 


\section{XII ${ }^{\text {èmes }}$ Journées Nationales Génie Côtier - Génie Civil \\ Cherbourg, 12-14 juin 2012}

exploitation dans le monde) permettra aux systèmes les plus performants de concurrencer les coûts complets de production d'un nombre significatif de systèmes commerciaux.

Le houlomoteur présente à court terme des fondamentaux économiques du même ordre que l'hydrolien, avec un coût du MW estimé entre 4 et $5 \mathrm{M} €$ au démarrage du marché. Le foisonnement technologique actuel et le manque de visibilité sur les systèmes gagnants diminuent le potentiel de réduction des coûts d'investissement à court terme, en retardant la mise en œuvre de procédures d'optimisation industrielle. On s'attend donc à un coût complet de production de l'ordre de 150 €/MWh à l'horizon 2020. A plus long terme, après la levée des incertitudes technologiques permettant d'exploiter la ressource stratégique plus éloignée des côtes -faisant référence à un potentiel de capacité installée gigantesque de l'ordre du TW, on anticipe une pénétration massive du houlomoteur telle que les économies d'échelle réalisées permettront d'atteindre un coût complet de production inférieur à $100 € / \mathrm{MWh}$.

Le coût complet de production d'une centrale ETM commerciale est évalué aujourd'hui par le monde industriel à $400 € / M W h$. Sur la base de telles hypothèses, l'ETM serait d'ores et déjà économiquement viable dans un ensemble d'îles tropicales dont les coûts complets de production électrique par centrales thermiques peuvent atteindre jusqu'à $700 € /$ MWh. L'accroissement des puissances nominales des centrales (passant de 10 à 50MW, voire 100MW) et les économies d'échelle réalisées contribueront à la réduction des coûts d'investissement et donc des coûts complets de production. Que ce soit à court terme via une subvention directe à l'investissement ou après 2025 par l'optimisation attendue des procédés industriels, un coût d'investissement d'environ $10 \mathrm{M€} / \mathrm{MW}$ (correspondant à un coût complet de production de $250 € / \mathrm{MWh}$ ) permettra la diffusion de la technologie ETM au sein de la centaine d'îles tropicales disposant d'une ressource éligible relativement proche des côtes.

\section{Synthèse et principaux éléments de conclusion sur la prospective des énergies marines à court, moyen et long termes}

La visibilité pour les investisseurs des gains potentiels de compétitivité et de l'évolution attendue des coûts sur le cycle complet des projets constituent des critères d'arbitrage de premier ordre : l'analyse de l'évolution des coûts complets de production des systèmes est décisive pour mesurer l'attractivité globale des énergies marines. Elle intègre, en plus des critères purement économiques (coût du MW, taux d'actualisation, coût d'exploitation), plusieurs variables sensibles, comme la performance des systèmes, ou encore la durée de vie des installations.

Le potentiel d'exploitation de la ressource en mer est tel qu'on envisage à terme sur le marché des énergies marines des investissements cumulés au niveau mondial de plusieurs milliers de milliards d'euros, correspondant à des capacités cumulées installés 
de l'ordre du millier de GW (ou TW), en phase avec la criticité que représente le levier des énergies renouvelables dans l'équation climatique (figure 2).

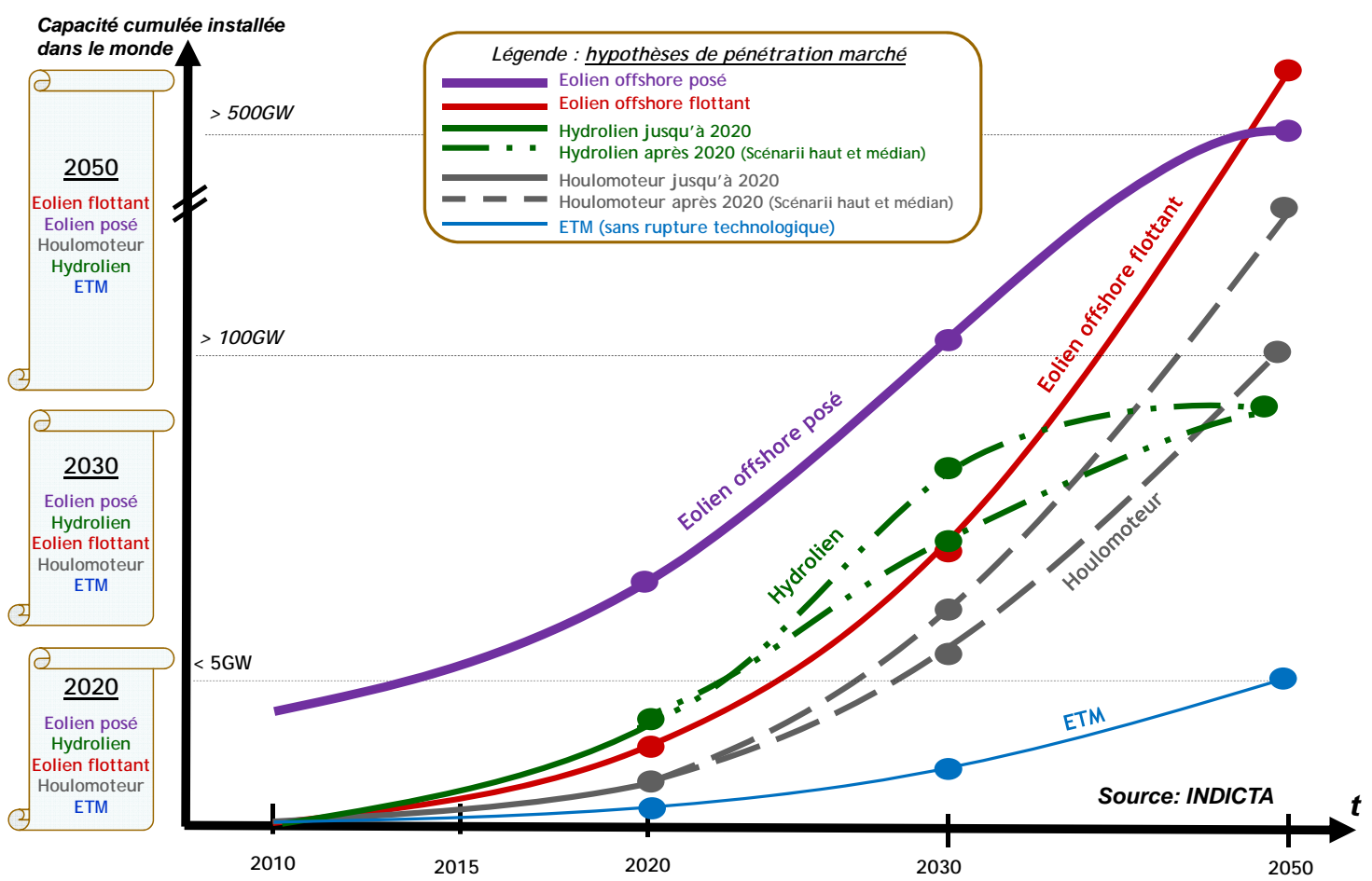

Figure 2. Prospective de marchés des cinq segments stratégiques des énergies marinesPérimètre mondial et horizon 2050.

La maturité des technologies, le degré de complexité des systèmes et enfin l'étendue de la ressource identifiée comme techniquement exploitable, conditionnent l'intérêt des industriels pour telle ou telle technologie ; de ce point de vue, l'éolien offshore (posé et flottant) ainsi que l’hydrolien doivent être considérés comme les marchés offrant les perspectives de croissance les plus importantes dans un horizon de vingt ans. L'ETM sera concentrée sur les zones intertropicales, et le houlomoteur constituera un marché stratégique sur le long terme.

\section{Références bibliographiques}

PAILLARD M., LAMBLIN V., LACROIX D., Coordination éditoriale (2007). Energies Marines Renouvelables, Etude prospective à l'horizon 2030, Editions Quae. [URL http://wwz.ifremer.fr/cop/content/download/15287/229854/file/Ifremer_syntheseetude-prospective-EnRM.pdf].

IPANEMA (2009). Initiative PArtenariale Nationale pour l'émergence des Energies MArines, rapport d'étape.

[URL http://www.ipanema2008.fr/images/stories/rapport_etape_ipanema_nov09.pdf]. 